\title{
Swelling behavior of hydrophobic polymers in water/ethanol mixtures
}

\author{
Wen-Yuan Chuang ${ }^{a}$, Tai-Horng Young ${ }^{\mathrm{a}, *}$, Da-Ming Wang ${ }^{\mathrm{b}}$, Ruei-Liang Luo ${ }^{\mathrm{c}}$, Yi-Ming Sun $^{\mathrm{c}}$ \\ ${ }^{a}$ Institute of Biomedical Engineering, College of Medicine and College of Engineering, National Taiwan University, Taipei 10016, Taiwan, ROC \\ ${ }^{\mathrm{b}}$ Department of Chemical Engineering, College of Engineering, National Taiwan University, Taipei 10016, Taiwan, ROC \\ ${ }^{\mathrm{c}}$ Department of Chemical Engineering, Yuan-Ze University, Chung-Li, Taoyuan, Taiwan, ROC
}

Received 22 December 1999; received in revised form 24 February 2000; accepted 29 February 2000

\begin{abstract}
Swelling behavior of poly(ethylene-co-vinyl alcohol) (EVAL), polyurethane (PU) and poly(ethylene-co-vinyl acetate) (EVAc) in ethanol/ water mixtures was investigated. In the case of EVAL, the total swelling curve rises to a maximum that is above the swelling of either pure constituent. On the other hand, in the PU and EVAc systems, the total swelling is intermediate between the swelling of the two pure components. In the case of PU, the total swelling is close to the ideal system as the water-rich region is approached. However, in the case of EVAc, the deviation from ideal is significantly negative. Theoretical swelling values have been derived from Flory-Huggins thermodynamics by using the equilibrium sorption data of the pure component. The theoretical results show good agreement with the swelling behavior of PU and EVAc in ethanol/water mixtures. However, the agreement between theoretical and experimental swelling values for EVAL is reasonably established when the Flory-Huggins equation was modified by incorporating a ternary interaction parameter $\chi_{\mathrm{T}}$. This is probably due to the fact that the water/ethanol mixture produces the water-ethanol complex that has a greater affinity for EVAL. In addition, the experimental results indicate that the shape of hydrophobic polymer swelling curve in the water/ethanol mixture is mainly controlled by the interactions between water and the polymer. Therefore, the correlation among the swelling equilibrium data, the polymer hydrophobicity and the structuring of water around the hydrophobic polymers is discussed. It is concluded that the hydrophobic interactions between the polymer and water are a major factor to influence the polymer swelling due to changes in the structuring of water around the hydrophobic polymers. These results might have important implications for the drug delivery and pervaporation, since these processes are influenced by the polymer swelling to a great degree. (C) 2000 Elsevier Science Ltd. All rights reserved.
\end{abstract}

Keywords: Swelling; Ternary interaction parameter; Water structure

\section{Introduction}

The equilibrium degree of swelling is an important property of a membrane, influencing as it does the permeability and mechanical properties. Especially, the knowledge gained from such investigation between a polymer and water/ethanol mixture would be of considerable interest to many applications of membranes, including drug delivery and pervaporation. The membrane-controlled reservoir system is one of the major types of drug-controlled-release devices [1]. In such a system, the water/ethanol mixture generally used for dissolving drugs might be able to swell the rate-controlling membrane and then alter the permeability of drugs through the membrane. Peppas and Reinhart [2], and Chen and Lostritto [3] have described the effect of swelling on the drug permeability through polymeric

\footnotetext{
* Corresponding author. Tel.: + 886-2-2397-0800, ext. 1455; fax: + 886-2-2394-0049.

E-mail address: thyoung@ha.mc.ntu.edu.tw (T.-H. Young).
}

membranes. In the case of pervaporation where a membrane separates a mixture of liquids, there has been considerable interest in the separation of water/ethanol mixtures. In fact, it is a well-established route to produce absolute ethanol by removing water from the water/ethanol mixture. In pervaporation, diffusion alone does not describe the transport process across a membrane sufficiently because the solubility of the permeant molecule in the membrane is a determining factor for the selectivity process as well. Similar to drug-controlled-release devices, the degree of membrane swelling has a tremendous influence on the diffusivity, making the polymer chains more flexible and resulting in an increased permeability. If an even greater interaction between liquid and membrane occurs, it will result in a much greater swelling of the polymer which allows relatively large molecules to diffuse through this kind of membrane. Thus the membrane swelling, as a result of interaction between the penetrant and the polymer, is a very important factor in transport through membranes. 
It is the main aim of the present work to perform experimental investigation on the effect of the water/ethanol mixture on the swelling of the polymeric membrane. It is well known that the water molecule is easily incorporated into the hydrophilic polymer membranes due to the strong affinity between the water molecule and the hydrophilic polymers. This generally leads to the excess swelling of the polymers, and results in unfavorable drug release rate or low membrane selectivity in pervaporation. Therefore, the following hydrophobic polymers have been studied: poly(ethylene-co-vinyl alcohol) (EVAL), polyurethane (PU) and poly(ethylene-co-vinyl acetate) (EVAc).

Analogous to Henry's law for the solubility of a gas in a liquid being directly proportional to the pressure of the gas above the liquid, the description of a liquid-liquid-polymer system with limited swelling can be given, which involves dilation of the polymer matrix to accommodate the absorbed liquids. Two absorbed liquids are then in equilibrium throughout the polymer. Therefore, the equilibrium swelling measures the tendency of molecules to partition from the liquid mixture into the polymer. At constant temperature, the equilibrium swelling can be classified into three general types.

Type I. The sorption isotherm is linear, i.e. the liquid composition inside the polymer is proportional to the external liquid composition. Such a case can be considered as an ideal system where the sorption of solvent in polymers is comparable to the Henry's law. Two liquids with closely similar properties, in which the interaction between unlike and like molecules are virtually identical, may follow this ideal solution behavior.

Type II. If the attractions between two liquid molecules are stronger than the attractions between the liquid molecule and the polymer, then adding one liquid to the other actually decreases the chance of the liquid molecule's partition within the polymer, and lowers the total swelling. Because the total swelling is lowered, this behavior is termed negative deviation.

Type III. The total swelling is above the swelling of either pure constituent. In this case, unlike liquid molecules may interact with one another less than the liquid molecule and the polymer do. Because the total swelling is increased relative to the ideal behavior, this behavior is termed positive deviation.

Clearly, the swelling behavior of hydrophobic polymer in water/ethanol mixtures is very complex because the liquid molecule in the polymer is far from being randomly distributed as a result of the interaction between the liquid molecule and the polymer. Thus, another objective of this paper is to describe and predict the complex swelling behavior on the basis of a suitable thermodynamic model. Theoretical values of polymer swelling have been derived from the Flory-Huggins thermodynamics [4]. To improve the agreement between experimental and theoretical data on polymer swelling Kargupta et al. [5] considered the binary inter- action parameters to be concentration dependent. Pouchly and Zivny [6] introduced a ternary parameter. In this paper we will follow the latter approach. The equations derived for the prediction of the theoretical swelling curves are based on the Flory-Huggins theory modified with a ternary interaction parameter, $\chi_{\mathrm{T}}$. We found the Flory-Huggins theory with a zero value of the ternary interaction parameter could correctly predict the swelling behavior of PU and EVAc in water/ethanol mixtures. However, in the waterethanol-EVAL system, no good agreement was found between the theoretical predictions and the experimentally obtained swelling data. Therefore, we discussed the EVAL swelling by a nonzero value of the ternary interaction parameter. In addition, the experimental results indicate that the shape of hydrophobic polymer-swelling curve in water/ ethanol mixture is mainly controlled by the interactions between water and the polymer. Hence, the correlation among the swelling equilibrium data, the polymer hydrophobicity and the structuring of water around the hydrophobic polymers is discussed.

\section{Experimental section}

\subsection{Material}

EVAL was obtained from Kuraray Co. Ltd, Japan (EPE105A) having an average ethylene content of $44 \mathrm{~mol} \%$. EVAc was obtained from E.I. Dupont de Nemours \& Co., Wilmington, DE (ELVAX-170) having an average ethylene content of $63 \mathrm{wt} . \%$. PU used in this study was synthesized by 4,4-dicyclohexylmethane diisocyanate (H12MDI, Mobay Co.), hydroxyl-terminated polybutadiene (HTPB, ARCO Co.) and 1,2-butanediol (Merck Co.) under the catalysis of dibutylzinn-dilaurat (DBTDL, Merck Co.). On can refer to the work of Huang and Lai [7] for more details of the synthesis process. Freshly opened absolute ethanol from USI (Tuscola, IL) was used. Water was deionized and double distilled before use.

\subsection{Methods}

For swelling measurements, dense membranes prepared by solvent evaporation were used. A piece of a known weight membrane was immersed into a flask containing the water/ethanol mixture of known composition that was present in large excess compared to the amount of membrane. Therefore, it allows determination of the swollen membrane being in equilibrium with a given composition of the liquid mixture. The membrane was then removed and weighed after the superfluous liquid was carefully wiped with tissue paper. The swelling equilibrium was established until no further weight increase was observed. Results of the water/ethanol mixture in EVAc were taken from Ref. [8]. The swelling degree has been expressed as a relative weight increase ( $\mathrm{g}$ of liquid/g of dry polymer). 


\subsection{Theory}

In this study, the Flory-Huggins theory is used to describe the state of equilibrium swelling of a membrane comprising two liquids with this two-liquid mixture, in which a ternary interaction parameter, $\chi_{\mathrm{T}}$, is included [9,10]:

$$
\begin{aligned}
\Delta G_{\mathrm{m}}= & \mathrm{R} T\left(n_{1} \ln \phi_{1}+n_{2} \ln \phi_{2}+n_{3} \ln \phi_{3}+\chi_{12} n_{1} \phi_{2}\right. \\
& \left.+\chi_{13} n_{1} \phi_{3}+\chi_{23} n_{2} \phi_{3}+\chi_{\mathrm{T}} n_{1} \phi_{2} \phi_{3}\right)
\end{aligned}
$$

where $n_{i}$ is the mole of the component $i, \phi_{i}$ its volume fraction and $\chi_{i j}$ the binary interaction parameter between the components $i$ and $j$. For the present ternary system, the subscript $i=1,2$ and 3 denotes water, ethanol and polymer, respectively. Since we investigated a membrane with limited swelling equilibrium, the contribution of the elastic free energy to the changes in the free energy of mixing was neglected. Differentiating Eq. (1) with respect to $n_{1}$ and $n_{2}$, the chemical potential, $\Delta \mu_{i}\left(\mu_{i}-\mu_{i}^{0}\right)$ in the membrane phase is given:

$$
\begin{aligned}
\frac{\Delta \mu_{1}}{\mathrm{R} T}= & \ln \phi_{1}+1-\phi_{1}-\frac{V_{1}}{V_{2}} \phi_{2}-\frac{V_{1}}{V_{3}} \phi_{3} \\
& +\left(\phi_{2} \chi_{12}+\phi_{3} \chi_{13}\right)\left(\phi_{2}+\phi_{3}\right)-\frac{V_{1}}{V_{2}} \phi_{2} \phi_{3} \chi_{23} \\
& -h_{1} h_{2} \phi_{2} \frac{\mathrm{d} \chi_{12}}{\mathrm{~d} h_{2}}-\phi_{1} \phi_{2} \phi_{3} \frac{\partial \chi_{13}}{\partial \phi_{2}}-\phi_{1} \phi_{3}^{2} \frac{\partial \chi_{13}}{\partial \phi_{2}} \\
& -\phi_{1} \phi_{3}^{2} \frac{\partial \chi_{13}}{\partial \phi_{3}}-\frac{V_{1}}{V_{2}} \phi_{2}^{2} \phi_{3} \frac{\partial \chi_{23}}{\partial \phi_{2}}-\frac{V_{1}}{V_{2}} \phi_{2} \phi_{3}^{2} \frac{\partial \chi_{23}}{\partial \phi_{3}} \\
& -\phi_{1} \phi_{2}^{2} \phi_{3} \frac{\partial \chi_{\mathrm{T}}}{\partial \phi_{2}}-\phi_{1} \phi_{2} \phi_{3}^{2} \frac{\partial \chi_{\mathrm{T}}}{\partial \phi_{3}}+\chi_{\mathrm{T}} \phi_{2} \phi_{3}\left(1-2 \phi_{1}\right)
\end{aligned}
$$

$$
\begin{aligned}
\frac{\Delta \mu_{2}}{\mathrm{R} T}= & \ln \phi_{2}+1-\phi_{2}-\frac{V_{2}}{V_{1}} \phi_{1}-\frac{V_{2}}{V_{3}} \phi_{3} \\
& +\left(\frac{V_{2}}{V_{1}} \phi_{1} \chi_{12}+\phi_{3} \chi_{23}\right)\left(\phi_{1}+\phi_{3}\right)-\frac{V_{2}}{V_{1}} \phi_{1} \phi_{3} \chi_{13} \\
& +\frac{V_{2}}{V_{1}} h_{1} h_{2} \phi_{1} \frac{\mathrm{d} \chi_{12}}{\mathrm{~d} h_{2}}+\frac{V_{2}}{V_{1}} \phi_{1} \phi_{3}\left(\phi_{1}+\phi_{3}\right) \frac{\partial \chi_{13}}{\partial \phi_{2}} \\
& -\frac{V_{2}}{V_{1}} \phi_{1} \phi_{3}^{2} \frac{\partial \chi_{13}}{\partial \phi_{3}}+\phi_{2} \phi_{3}\left(\phi_{1}+\phi_{3}\right) \frac{\partial \chi_{23}}{\partial \phi_{2}} \\
& -\phi_{2} \phi_{3}^{2} \frac{\partial \chi_{23}}{\partial \phi_{3}}+\frac{V_{2}}{V_{1}} \phi_{1} \phi_{2} \phi_{3}\left(\phi_{1}+\phi_{3}\right) \frac{\partial \chi_{\mathrm{T}}}{\partial \phi_{2}} \\
& -\frac{V_{2}}{V_{1}} \phi_{1} \phi_{2} \phi_{3}^{2} \frac{\partial \chi_{\mathrm{T}}}{\partial \phi_{3}}+\frac{V_{2}}{V_{1}} \chi_{\mathrm{T}} \phi_{1} \phi_{3}\left(1-2 \phi_{2}\right)
\end{aligned}
$$

where $\mu_{i}^{0}$ is the chemical potential of component $i$ at standard state, $V_{i}$ is the molar volume, $h_{1}=\phi_{1} /\left(\phi_{1}+\phi_{2}\right)$ and $h_{2}=\phi_{2} /\left(\phi_{1}+\phi_{2}\right)$. Likewise, the chemical potential,
$\Delta \mu_{i}$ in the liquid mixture phase can be obtained according to Eqs. (3) and (4) by assuming $\phi_{3}=0$.

At equilibrium between the liquid mixture phase and the membrane phase ( 1 and $\mathrm{m}$ ) at a specified temperature and pressure, the chemical potential of each liquid component in these two phases are equal, i.e.

$\mu_{1}^{1}=\mu_{1}^{\mathrm{m}}$

$\mu_{2}^{1}=\mu_{2}^{\mathrm{m}}$

where $\mu_{i}^{1}$ and $\mu_{i}^{\mathrm{m}}$ are the chemical potentials of component $i$ in the liquid mixture phase and the membrane phase, respectively. Since volume fractions of all components in the membrane phase $\left(\phi_{i}^{\mathrm{m}}\right)$ add up to 1 , we have:

$\phi_{1}^{\mathrm{m}}+\phi_{2}^{\mathrm{m}}+\phi_{3}^{\mathrm{m}}=1$

Eqs. (2)-(6) describe the compositions of the membrane phase at equilibrium. Given the interaction parameters and the molar volumes, these equations can be used to compute the theoretical membrane swelling $\left(\left(\rho_{1} \phi_{1}^{\mathrm{m}}+\right.\right.$ $\left.\rho_{2} \phi_{2}^{\mathrm{m}}\right) / \rho_{3} \phi_{3}^{\mathrm{m}}$ ) in this report, where $\rho_{i}$ is the density of component $i$.

\subsection{Interaction parameters}

The binary interaction parameters between water and ethanol, $\chi_{12}$, at 37 and $32^{\circ} \mathrm{C}$ are calculated from the excess Gibbs energy data using the group contribution method of UNIFAC [11]. Assuming that $\chi_{12}$ has the functional form suggested by Koningsveld and Kleintjens [12], parameters $a, b$ and $c$ in Eq. (8) can be found by leastsquare regression.

$\chi_{12}=a-\frac{b}{1-c \phi_{2}}$

For a ternary system, following Yilmaz and McHugh [13], $\chi_{12}$ is assumed to be a function only of $\phi_{2} /\left(\phi_{1}+\right.$ $\phi_{2}$ ). Thus, $\chi_{12}$ is obtained by replacing $\phi_{2}$ with $h_{2}=$ $\phi_{2} /\left(\phi_{1}+\phi_{2}\right)$ in Eq. (7).

Binary interaction parameters between pure liquid and polymer, $\chi_{13}$ and $\chi_{23}$, are considered to be concentration independent due to low sorption in this study. Thus, $\chi_{13}$ and $\chi_{23}$ can be determined using data from equilibrium swelling experiments of the pure components in the corresponding membrane [14]. The following equation is employed:

$\ln \phi_{i}+\left(1-\frac{1}{x_{n}}\right) \phi_{3}+\chi_{i 3} \phi_{3}^{2}=0 \quad i=1$ or 2

where $x_{n}$ is the degree of polymerization of the polymer.

Experimental data for the ternary interaction parameter, $\chi_{\mathrm{T}}$, is not available in literature. In this study, we simply treat $\chi_{\mathrm{T}}$ as an empirical correction parameter. A fitting procedure was performed by systematically varying $\chi_{\mathrm{T}}$ to see if the total membrane swelling fits the experimental data. It will be shown that there is a reasonable agreement 

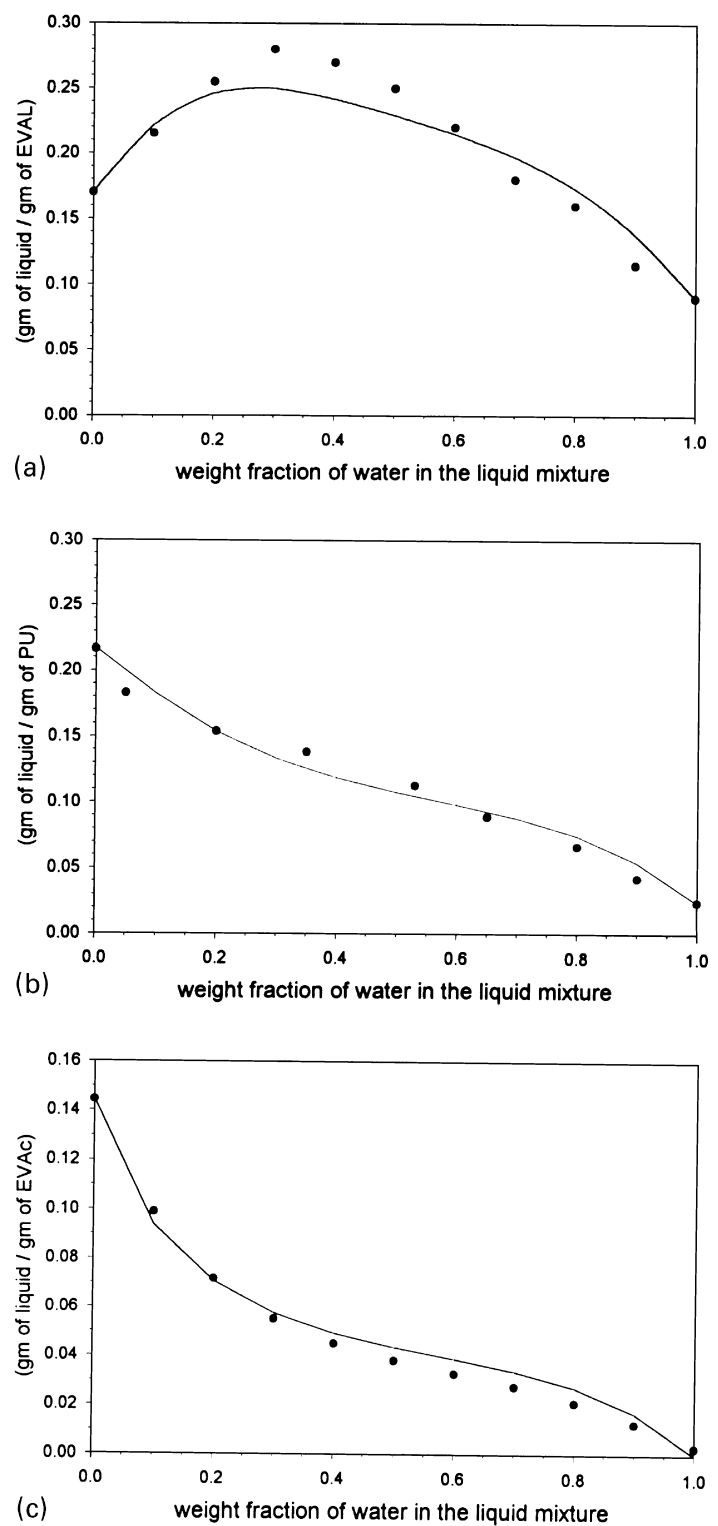

Fig. 1. Experimental data $(\bullet)$ and theoretical values according to the Flory-Huggins theory with $\chi_{\mathrm{T}}=0$ (-) for the total swelling of: (a) EVAL; (b) PU; and (c) EVAc in the water/ethanol mixtures as a function of the weight fraction of water in the liquid.

between the experimentally obtained and the theoretically predicted data at $\chi_{\mathrm{T}}=0$ in the PU and EVAc systems, but it cannot be obtained by simply using binodal interaction parameters only for the EVAL system.

Table 1

Physical properties of water, ethanol, EVAL, PU and EVAc

\begin{tabular}{lcl}
\hline Component & Molecular weight & Density $\left(\mathrm{g} / \mathrm{cm}^{3}\right)$ \\
\hline Water & 18 & 1 \\
Ethanol & 44 & 0.78 \\
EVAL & 56000 & 1.14 \\
PU & 70000 & 0.99 \\
EVAc & 48100 & 0.96
\end{tabular}

Table 2

Summary of binary interaction parameters

\begin{tabular}{ll}
\hline & $\chi_{i j}$ \\
\hline At $37^{\circ} \mathrm{C}$ & \\
Water-EVAL & 1.784 \\
Water-PU & 3.723 \\
Ethanol-EVAL & 1.274 \\
Ethanol-PU & 1.223 \\
Water-ethanol & $0.777+0.197 /\left(1.0-0.704 h_{2}\right)^{\mathrm{a}}$ \\
At $32^{\circ} \mathrm{C}$ & \\
Water-EVAc & 4.908 \\
Ethanol-EVAc & 1.451 \\
Water-ethanol & $0.786+0.191 /\left(1.0-0.706 h_{2}\right)^{\mathrm{a}}$ \\
\hline
\end{tabular}

${ }^{\mathrm{a}} h_{2}=\phi_{2} /\left(\phi_{1}+\phi_{2}\right)$.

\section{Results}

Fig. 1(a),(b) shows the experimental total swelling values of the water/ethanol mixture in EVAL and PU (overall liquid sorption per unit gram of dry polymer) at $37^{\circ} \mathrm{C}$. The results are presented as a function of the weight fraction of water in the liquid mixture. Swelling results for the water/ ethanol mixtures in EVAc at $32^{\circ} \mathrm{C}$ have already been published previously [8] and are shown in Fig. 1(c) for comparison. The data show that the sorption amount of pure water was smaller in comparison with pure ethanol for all of the polymers. This behavior is expected because all of the polymers studied are hydrophobic. However, the shape of the swelling curves is quite different for these polymers in the water/ethanol mixtures. In the case of EVAL, the polymer swelling exhibits positive deviation from ideal, which is above the swelling of either pure constituent. The swelling curve rises to a maximum at $30 \mathrm{wt} . \%$ of water in liquid mixtures. EVAc shows an opposite behavior. The deviation from ideal is negative but the swelling values are always intermediate between that of the two pure components. On the other hand, in the case of PU, the deviation from ideal is not evident. A tendency to an ideal, additive swelling behavior is observed. Similar swelling behavior has been observed by Mulder et al. [15] for cellulose acetate and polysulfone in water/ethanol mixtures.

\subsection{Prediction of swelling behavior}

In order to explain the difference in swelling behavior of hydrophobic polymers in water/ethanol mixtures, the equations derived in Section 2.3 were analyzed under various conditions. The parameters in the construction of theoretical equilibrium swelling curves are not arbitrarily selected; however, they are chosen to be very close to the typical experimental parameters. The physical constants required in the computations are given in Table 1 . The concentration-dependent interaction parameter for the water-ethanol binary pair, $\chi_{12}$, was calculated from the excess Gibbs energy data using the group contribution method of 


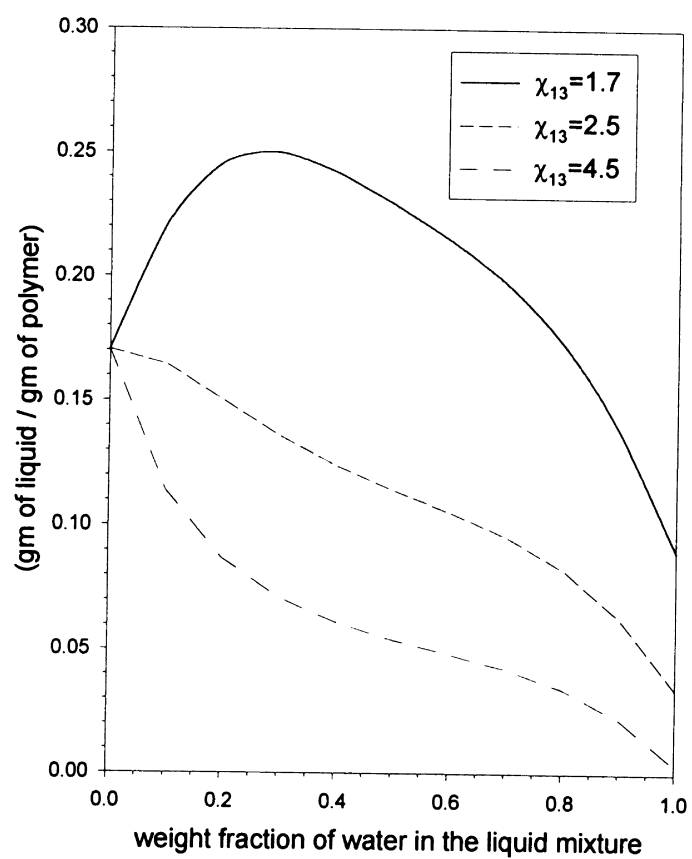

Fig. 2. Theoretical swelling curves of the hydrophobic polymers in the water/ethanol mixtures, where $\chi_{23}=1.27$ and $\chi_{13}=1.7,2.5$ and 4.5 , respectively.

UNIFAC [11]. Nonlinear regression was then used with Eq. (7) to find the parameters $a, b$ and $c$; the results are given in Table 2. At first, it was assumed that the value of $\chi_{\mathrm{T}}$ is zero, which means the ternary interaction parameter is neglected. The high $\chi_{13}$ values $(1.7,2.5$ and 4.5$)$ were used to calculate the swelling behavior due to the low interaction between water and the hydrophobic polymer. The $\chi_{23}$ value, 1.27, was taken to be close to the interaction parameter of ethanol-EVAL.

The influence of the affinity of water towards the hydrophobic polymer on the polymer swelling is given in Fig. 2. As shown in Eqs. (2)-(6), with $\chi_{\mathrm{T}}$ being zero, although the polymer swelling mainly depends on the affinity of both water and ethanol towards the polymer, it is obvious that the choice of $\chi_{13}$ values greatly affects the polymer swelling from these three numerical swelling curves. Clearly, the lower $\chi_{13}$ value gives a greater polymer swelling. In addition, the contribution of $\chi_{13}$ to the shape of the swelling curve is quite significant. The swelling curve is convex at $\chi_{13}=1.7$ and concave at $\chi_{13}=4.5$. At $\chi_{13}=2.5$ the deviation of the swelling curve from ideal is not evident.

In light of the above result the water-polymer interaction parameter significantly influences the swelling behavior for a polymer in the aqueous ethanol solution; thus, we further investigated extensively the effect of $\chi_{13}$ on the polymer swelling behavior. Fig. 3 shows another case of how a theoretical swelling curve may continuously change with $\chi_{13}$ from 1.5 to 5.0, still keeping $\chi_{23}$ at a constant but lower value (1.0). As expected an increase in the interaction parameter $\chi_{13}$ decreases the extent of swelling. If $\chi_{13}>2.7$, the swelling curve is concave and asymmetric for this system

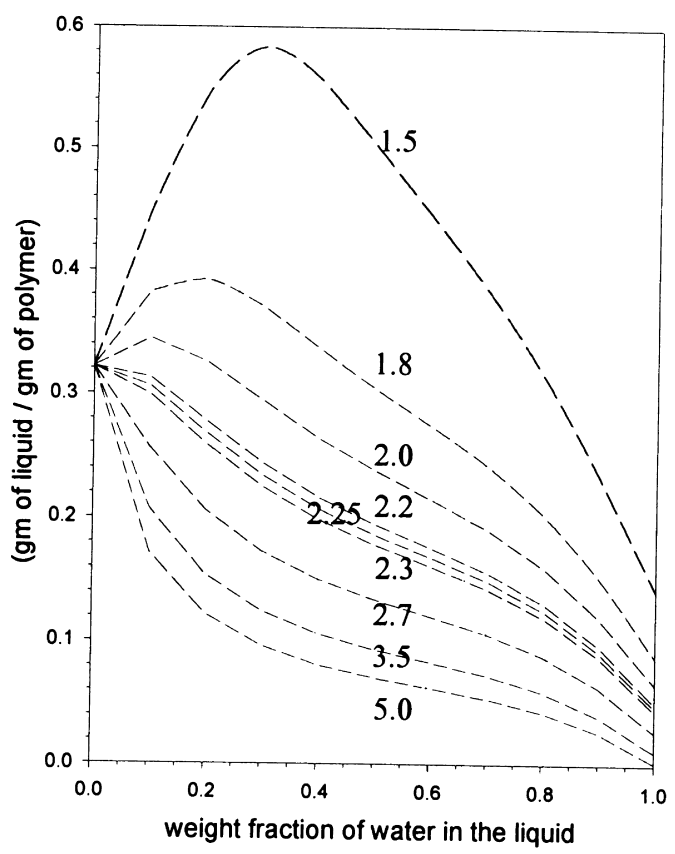

Fig. 3. Effect of $\chi_{13}$ on the swelling curves of the hydrophobic polymers in the water/ethanol mixtures at $\chi_{23}=1.0$. The numbers shown denote the value of $\chi_{13}$ used for the calculations.

with the weight fraction of water. As the $\chi_{13}$ value decreases, the polymer swelling relative to ideal gradually changes from a negative to a positive deviation. In other words, instead of concave curves, swelling patterns with convex shape are observed. The first maximum in the swelling curve appears when the $\chi_{13}$ value is approximately as small as 2.0. In addition, note that the maximum swelling does not occur at the same composition, the maximum swelling for $\chi_{13}=2.0$ occurs at $x=0.1$ and for $\chi_{13}=1.5$ at $x=$ 0.3 . These phenomena are similar to the experimental results of the hydrophobic polymers in the aqueous ethanol solution. Based on Figs. 2 and 3, the shape of the swelling curve is greatly influenced by $\chi_{13}$, even $\chi_{23}$ is changed to a smaller value. In the next section we will see how the experimental and theoretical data agree.

\subsection{Theoretical swelling behavior of EVAL, PU and EVAc}

It has been shown that the swelling equilibrium in the system polymer-mixed solvent can be derived from the Flory-Huggins theory with a number of parameters. Therefore, the theoretical swelling values for EVAL, PU and EVAc as a function of the weight fraction of water in aqueous ethanol solution determined by solving Eqs. (2)(6), together with the experimental data points for comparison, are given in Fig. 1. The physical constants for EVAL, PU and EVAc employed in the swelling computations are given in Table 1. The values of $\chi_{13}$ and $\chi_{23}$ were found using Eq. (8) from the equilibrium absorption of pure water and pure ethanol; the results are given in Table 2. Fig. 1(b),(c) shows that the results of the theoretical calculation with 


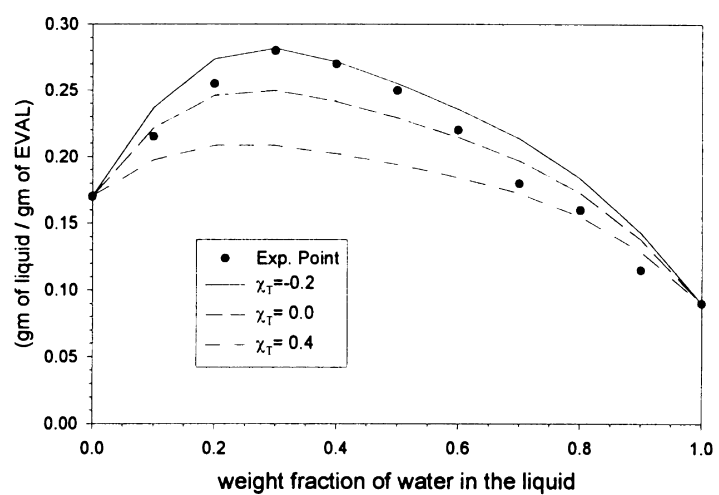

Fig. 4. Experimental data (O) and theoretical values with different $\chi_{\mathrm{T}}$ values for the total swelling of EVAL in the water/ethanol mixtures as a function of the weight fraction of water in the liquid.

$\chi_{\mathrm{T}}=0$ are in line with the experimental findings for PU and EVAc in the water/ethanol mixtures. However, in the EVAL system, although both the experimental and the theoretical swelling values with $\chi_{\mathrm{T}}=0$ show a similar trend, the theoretical values are not in good agreement with the experimental data over the entire composition range. The deviations of experimental data from calculation are positive in the ethanol-rich region, but the deviations are slightly negative in the water-rich region. Even if their maximum swelling values occur approximately at the same liquid composition, the maximum value of the experiment is larger than that of the calculated. This indicates that only the binary interaction parameter approaching the water-ethanol-EVAL system is inadequate. To improve the agreement between the experimental and theoretical values, one might attempt to rationalize this behavior to postulate the existence of a ternary interaction parameter, $\chi_{\mathrm{T}}$, to modify the interaction of EVAL with the water/ethanol mixture $[9,10]$. In Fig. 4 the experimental swelling values and the theoretical results with different $\chi_{\mathrm{T}}$ values for the total swelling of the water/ethanol mixtures in EVAL are given. Regardless

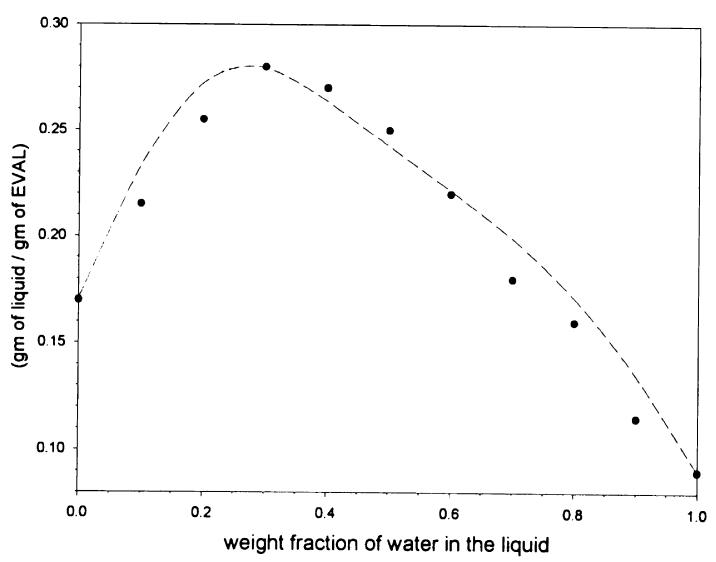

Fig. 5. Experimental data $(\bullet)$ and theoretical values using $\chi_{\mathrm{T}}=-1.0+$ $0.02 \phi_{2}+1.39 \phi_{3}$ (- - ) for the total swelling of EVAL in the water/ethanol mixtures as a function of the weight fraction of water in the liquid.
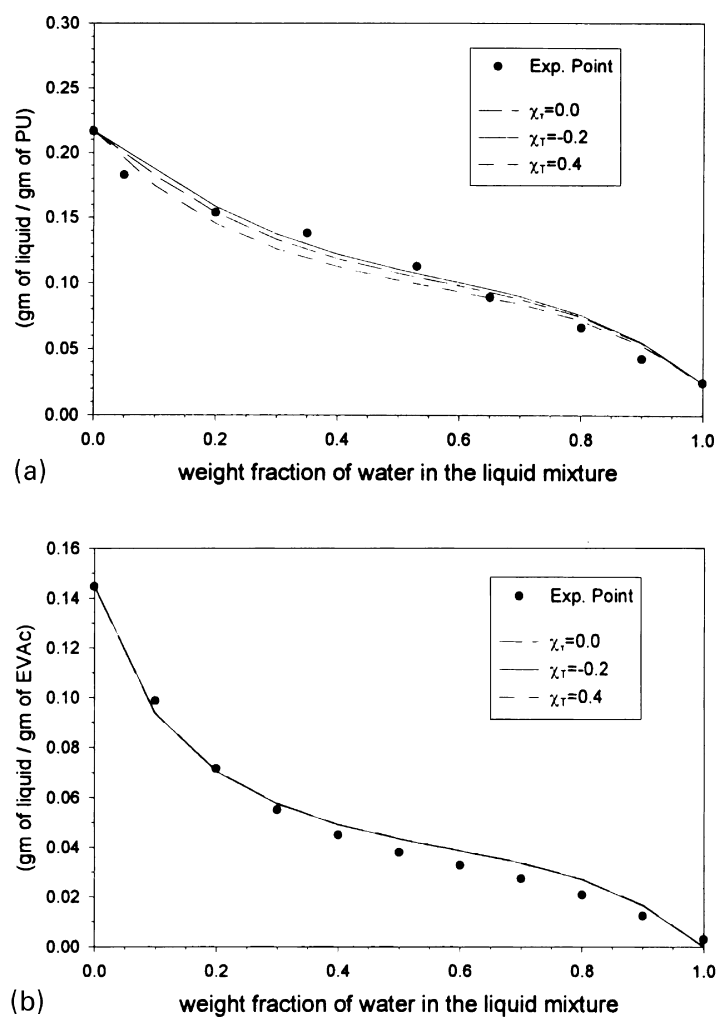

Fig. 6. Experimental data $(\bullet)$ and theoretical values with different $\chi_{\mathrm{T}}$ values for the total swelling of: (a) PU; and (b) EVAc in the water/ethanol mixtures as a function of the weight fraction of water in the liquid.

of the $\chi_{\mathrm{T}}$ value, all of the calculation results show that the degree of swelling increases to a maximum value and then decreases as the weight fraction of water in the liquid mixture is increased. Similar to the binary interaction parameter, the parameter $\chi_{\mathrm{T}}$ with a negative value has a positive effect on the polymer swelling. Conversely, taking a positive $\chi_{\mathrm{T}}$ value, the polymer swelling indicates a lower value. It is clear that a reasonable agreement between the calculation and the experiment is obtained by using $\chi_{\mathrm{T}}=0.4$ in the water-rich region, but it indicates a lower theoretical value compared to the experimental data for a liquid composition with less than $70 \mathrm{wt} . \%$ water in liquid mixtures. In contrast, when the parameter $\chi_{\mathrm{T}}$ is -0.2 , the maximum swelling value occurring at $30 \mathrm{wt} . \%$ water in liquid mixtures is fitted, whereas for concentrations having more than $50 \mathrm{wt} . \%$ water in liquid mixtures, an overestimation of polymer swelling is found. Since $\chi_{\mathrm{T}}$ varies with liquid composition and alters the swelling degree considerably, it was further attempted to see if it would be possible to fit the experimental data by using a concentration-dependent $\chi_{\mathrm{T}}$. The role of concentration-dependent $\chi_{\mathrm{T}}$ in the EVAL-water-2-propanol system at $60^{\circ} \mathrm{C}$ has been investigated in a previous publication [10]. It could be shown that in the Flory-Huggins theory with inserted $\chi_{\mathrm{T}}$ two nonsolvents have the character of a cosolvent. In the present study, the water-ethanol-EVAL system is also a perfect example to demonstrate the presence of a "cosolvent". For a pure liquid low sorption is observed; 
however, EVAL appears to be highly swelled in the water/ ethanol mixtures. A trial-and-error procedure was used to obtain the concentration-dependent $\chi_{\mathrm{T}}$, in which experimental data points on the swelling curve were fitted. A three-parameter rational form of $\chi_{\mathrm{T}}$ made by Young et al. [10] was followed. The best fit is obtained by using $\chi_{\mathrm{T}}=$ $-1.0+0.02 \phi_{2}+1.3 \phi_{3}$. Fig. 5 shows that the computed swelling values match closely the experimental data points over the entire composition range, suggesting that a reliable curve fitting has been obtained. Therefore, it is reasonable to introduce a ternary interaction parameter when binary parameters provide an inadequate description of swelling behavior.

For comparison, the experimental swelling values and the theoretical results, with different $\chi_{\mathrm{T}}$ values for the total swelling of water/ethanol mixtures in PU and EVAc, are given in Fig. 6(a),(b). It can be seen that the PU system gives a slightly different or the same swelling results. Furthermore, three sets of swelling data are virtually the same in the EVAc system, which implies that the contribution of the ternary interaction parameter is insignificant or can be ignored in these two systems. This is probably caused by the fact that the formation of the so-called waterethanol-polymer contacts is difficult [10], because of very limited water sorbed in such strongly hydrophobic polymers. This suggests that only the binary interaction parameters are enough to describe the PU and EVAc systems.

\section{Discussion}

Polymer swelling is a thermodynamic phenomenon, and it gives a measure of the amount of liquid sorbed by the polymer under equilibrium conditions. For ideal systems, the sorption isotherm is assumed to be linear (Henry's law), i.e. the liquid concentration inside the polymer is proportional to that outside. However, for the systems studied, the swelling curve is curved rather than linear. Thus, the assumption of ideal sorption behavior cannot be used in the present study.

Within the context of this paper the Flory-Huggins theory has been tired to be used to describe the nonideal swelling behavior of the hydrophobic polymers in the ethanol/water mixtures. Based on our results, the water-ethanol-hydrophobic polymer system is very interesting because three types of polymer swelling from the water/ ethanol mixtures may occur; it is shown that the swelling pattern can be appropriately predicted. In particular, the theoretical results show a good agreement with the experimental data of PU and EVAc. Although no good agreement was found in the EVAL system, an improvement can be obtained when a ternary interaction parameter is considered. In addition, the shape of the swelling curve can be ascribed to the affinity of the pure component towards the polymer. Especially, based on Figs. 2 and 3, the mutual affinity between water and the polymer contributes to a large extent to the shape of the swelling curve. Therefore, when the parameter $\chi_{12}$ is fixed for the ethanol/water mixtures, the parameter $\chi_{13}$ almost dominates the swelling behavior of the polymer in the ethanol aqueous solution.

Although a reasonable agreement can be obtained between the theory and the experiment, a question about the physical interpretation of this study, however, remained to be solved. What is the mechanism to influence the swelling degree of the hydrophobic polymers in the water/ ethanol mixtures? Basically, the component with smaller volume will be sorbed to a greater degree. The molar volume of water is smaller compared to ethanol but water molecules will group together to organize into clusters due to the formation of hydrogen bonds among the water molecules according to the flickering-cluster model of Frank and Wen [16]. Therefore, the lower equilibrium absorption of water in the polymer is due to the presence of associated forms, i.e. the component penetrates not as a single molecule but in its clustered form. This implies that the size of the component increases and that the number of the penetrant molecules consequently decreases. In addition, the equilibrium between the isolated water molecules and the associated water molecules influenced by other surrounding molecules is important, especially those via hydrogen bonding. Hence, the effect of ethanol on water structure cannot be neglected because ethanol can participate in hydrogen bonds. In fact, heat evolution and a large decrease in entropy are observed when water is mixed with ethanol, suggesting the mixing process will lead to degradation of the water clusters and to the formation of more and better hydrogen bonds in the mixtures as compared with either liquid alone. Furthermore, the interaction between the polymer and water must be understood if we are to achieve detailed understanding of the polymer swelling applied to the aqueous solution. When the polymer is added to water, the water cluster structure will be enhanced or demolished depending on the nature of interactions. In the presence of the hydrophobic polymer, it will shift the unassociated water molecules to larger clusters around the hydrophobic polymer due to repulsion by the exposure of hydrophobic chains to water in such a manner that the unassociated water molecules further group together to enhance the overall structural order. Hence, in all the systems studied here, the balance of ethanol and the polymer to enhance and disturb the flickering clusters is discussed.

\subsection{The water-ethanol-EVAc system}

Based on the above discussion, water molecules experience strong hydrogen bonding, which means that the water molecules may penetrate the polymer, accompanied by the clustered molecules. The extent of clustering will depend on the type of the polymer and other penetrant molecules present. In the case of EVAc, the water molecules in the solution around EVAc are far from being randomly distributed as a result of the hydrophobic interaction between the 
water molecules and EVAc. Therefore, very limited water sorbed in such a strongly hydrophobic polymer (Fig. 1(c)) is primarily attributed to more structured water clusters surrounding the polymer due to the hydrophobic hydration. Further, when ethanol is added to water, the ethanol molecules like to stay in the solution due to the strong interaction between ethanol and water. Therefore, the restriction of ethanol molecules will decrease the EVAc swelling. The decrease of the EVAc swelling implies that the intramolecular hydrophobic interactions of the ethylene groups are increased, which in turn may promote further shrinkage of EVAc. Hence, the total swelling is always negative from ideal. As a consequence the effect of the hydrophobic nature on the polymer swelling contributes to a greater degree if the polymer hydrophobicity is as strong as that of EVAc.

\subsection{The water-ethanol-PU system}

As was found for the EVAc system, polymer swelling lower than ideal should be expected in the water-ethanol-PU system. However, the difference between PU and the ideal swelling becomes smaller and only occurs in the ethanol-rich region. Even in the water-rich region, PU gives the same or slightly larger swelling than ideal. This behavior could be expected because of the higher sorption amount of pure water in PU relative to in EVAc (see Fig. 1). Thus, the structural order of the water clusters around PU is not as complete as around EVAc. In addition, it has been suggested by Soper and Finney [17] that water molecules form a disordered tetrahedral structure around an alcohol molecule. Hence, in the presence of EVAc, the structural order of water clusters is not changed when ethanol is added to water. However, as PU is present, ethanol molecules disturb the water clusters so as to swell the polymer. Overall, when the polymer can enhance the structured water cluster it will decrease the polymer's swelling degree. Conversely, when ethanol can destroy the structured water cluster it will increase the polymer's swelling degree.

\subsection{The water-ethanol-EVAL system}

The negative deviation from ideal for the EVAL swelling in the water/ethanol mixtures was not observed, even above the swelling of either pure constituent (Fig. 1). This behavior could already be expected because of the higher affinity between water and EVAL in comparison with water and PU. Therefore, as the polymer hydrophobicity decreases, ethanol can disturb the structured water cluster to increase the polymer swelling in the water/ethanol mixture. In addition, it is evident from Fig. 1(a) that the measured maximum swelling value is larger than that of the calculation with $\chi_{\mathrm{T}}=0$. The EVAL swelling implies that the intramolecular hydrogen bonding between the vinyl alcohol groups and the hydrophobic interactions of the ethylene groups are decreased. It appears as though the water/ethanol mixture produces the water-ethanol complex that has a greater affinity for EVAL. It can be thought that the ethanol molecules fit into the space between the water clusters to break down the structural order of the water clusters to form the waterethanol complex. Then the ethanol molecules show a favorite orientation wherein the hydrophobic parts of the ethanol molecules pointed in the direction of the polymer and the hydrophilic parts pointed in the direction of the water molecules [18]. Therefore, the water-ethanol complex can form a hydrogen bond with the vinyl alcohol groups and can form a hydrophobic interaction with the ethylene groups, which in turn may promote further swelling of EVAL. In fact, EVAL is insoluble in either water or ethanol separately but certain mixtures of water and ethanol dissolve EVAL at $60^{\circ} \mathrm{C}$ (data not shown). Accordingly, it can be proposed that this system favors the formation of water-ethanolEVAL contacts than water-EVAL and ethanol-EVAL contacts. In this circumstance, only binary interaction parameters approaching the EVAL swelling are inadequate, i.e. the ternary interaction cannot be neglected.

\section{Conclusion}

The measurements have been performed by swelling hydrophobic membrane samples in water/ethanol mixtures. The complex formations discussed above will be further investigated through additional experiments such as calorimetry, IR and Raman spectroscopy in the future. From the current work the following conclusions can be made:

1. The assumption of an ideal sorption behavior cannot be used for the water-ethanol-hydrophobic polymer systems. However, the results presented here demonstrate clearly that it is possible to predict the trend of membrane swelling characteristics for nonideal mixtures like water/ ethanol from a single-component sorption only.

2. Although the $\chi_{\mathrm{T}}$ value is only an estimate, our study indicates that $\chi_{\mathrm{T}}$ is a concentration-dependent parameter, and it might even describe the microscopic behavior of the water-ethanol-EVAL system to coincide with the experimental data.

3 . The changes in the polymer swelling are due to changes in the polymer hydrophobicity. This suggests that the interactions between the polymer and water are a major factor in determining the polymer swelling caused by changes in structuring of water around hydrophobic polymers.

\section{Acknowledgements}

The authors thank the National Science Council of the Republic of China for their financial support of project NSC 89-2216-E-002-006.

\section{References}

[1] Baker RW. Controlled release of biologically active agents. New York: Wiley, 1987.

[2] Peppas NA, Reinhart CT. J Membr Sci 1983;15:275. 
[3] Chen SX, Lostritto RT. J Controlled Release 1996;38:185.

[4] Flory P. Principles of polymer chemistry. Ithaca, NY: Cornell University Press, 1953.

[5] Kargupta K, Datta S, Sanyal SK. J Membr Sci 1997;124:253.

[6] Pouchly J, Zivny A. Makromol Chem 1982;183:3019.

[7] Huang SL, Lai JY. J Membr Sci 1995;105:137.

[8] Dinh SM, Berner B, Sun YM, Lee PI. J Membr Sci 1992;69:223.

[9] Van de Witte P, Dijkstra PJ, Van de Berg JWA, Feijen J. J Polym Sci Polym Phys 1996;34:2553.

[10] Young TH, Cheng LP, Hsieh CC, Chen LW. Macromolecules 1998;31:1229.
[11] Frednslund A, Gmehling J, Michelsen L, Rasmussen P, Prausnitz JM. Ind Engng Chem Process Des Dev 1977;16:450.

[12] Koningsveld R, Kleintjens LA. Macromolecules 1971;4:637.

[13] Yilmaz L, McHugh AJ. J Appl Polym Sci 1986;31:997.

[14] Bonner D. J Macromol Sci Rev Macromol Chem 1975;13:263.

[15] Mulder MHV, Franken T, Smolders CA. J Membr Sci 1985;22:155.

[16] Frank H, Wen W. Disc Faraday Soc 1957;24:133.

[17] Soper AK, Finney JL. Phys Rev Lett 1993;27:4346.

[18] Fritz L, Hofmann D. Polymer 1998;39:2531. 